\title{
La caverna iluminada: una singular lámpara gravetiense arroja luz sobre el arte parietal de la cueva de La Pileta (Benaoján, Málaga)
}

\author{
The illuminated cavern: a singular Gravettian lamp throws light upon the rock art at the cave \\ of La Pileta (Benaoján, Málaga)
}

\begin{abstract}
Miguel Cortés Sánchez ${ }^{a}$, María Dolores Simón Vallejo ${ }^{b}$, Arturo Morales-Muñiz ${ }^{c}$, $\mathbf{M}^{\mathrm{a}}$ Carmen Lozano Francisco ${ }^{d}$, José L. Vera Peláez ${ }^{\mathrm{d}}$ y Carlos Odriozola Lloret ${ }^{\mathrm{a}}$
\end{abstract}

\section{RESUMEN}

En este trabajo presentamos el estudio arqueológico, paleontológico, tafonómico, cronológico y geoquímico de una valva de ostra hallada en la cueva de La Pileta. El ejemplar procede de depósitos fosilíferos neógenos distantes más de $10 \mathrm{~km}$ del yacimiento. Una vez adaptada, la concha fue empleada como lámpara portátil. La cronología ${ }^{14} \mathrm{C} / \mathrm{AMS}$ de la costra carbonatada que sellaba la cubeta nos remite al periodo Gravetiense. Según diversos indicios en la lámpara, durante su vida útil se procesaron o almacenaron pigmentos. Los datos aportados informan sobre la dispersión del simbolismo del Paleolítico Superior Inicial hasta el extremo sudoccidental europeo, así mismo, suma nuevos elementos al debate sobre la existencia de una fase presolutrense en el arte parietal de La Pileta y, por ende, de todo el sur de la Península Ibérica. cate that during its use life, the valve was used to harbor or process pigments. The data presented throw light on the spread of symbolism in the southwestern corner of Europe during the Early Upper Palaeolithic, and add elements to the debate on the existence of a pre-Solutrean stage in the rock art of La Pileta and, by extension, of the southern Iberian Peninsula.

Palabras clave: Fósil; Lámpara; Gravetiense; Arte paleolítico; Península Ibérica.

Key words: Fossil; Lamp; Gravettian; Paleolithic Rock Art; Iberian Peninsula.

\section{INTRODUCCIÓN}

La Asamblea General de Naciones Unidas declaró 2015 "Año Internacional de la Luz y las Tecnologías Basadas en la Luz" (A/RES/68/221), como reconocimiento de la importancia que la luz tiene en la existencia humana. El primer hito en la creación de la luz artificial estuvo ligado a la generalización del control del fuego, fundamentalmente, durante el Pleistoceno medio avanzado a Gravettian chronology. Different lines of evidence indi-

a Dpto de Prehistoria y Arqueología, Facultad de Geografía e Historia. Universidad de Sevilla. C/ Doña María de Padilla s/n. 41004. Sevilla. Correos e.: mcortes@us.es; codriozola@us.es

b Museo Arqueológico de Frigiliana. C/ Cuesta del Apero 10.29788 Frigiliana. Málaga. Correo e.: simonmd63@gmail.com

c Laboratorio de Zooarqueología, Dpto de Biología. Universidad Autónoma de Madrid. 28049 Madrid. Correo e.: arturo.morales@uam.es

d Grupo HUM-949. Tellus. Prehistoria y Arqueología en el sur de Iberia. Correo e.: joselverapelaez@gmail.com

Recibido 14-V-2015; aceptado 1-IX-2015. 
(p. ej. Bolomor o Ángel para la península ibérica, Fernández et al. 2012; Monge et al. 2014 con discusión de contextos más antiguos). El segundo podríamos vincularlo al control de su portabilidad. Los primeros indicios arqueológicos de este último desarrollo serían los datos directos de antorchas y lámparas hallados en lugares recónditos, como cavernas (Beaune 1987), o indirectos, como evidencias arqueológicas de circulación humana por zonas de total oscuridad (icnitas, restos arqueológicos o antropológicos depositados intencionalmente, etc.). En la península ibérica, las primeras lámparas portátiles se encuadran en el Paleolítico Superior (Tab. 1) y, básicamente, proceden de la cornisa cantábrica (Rasilla et al. 2010). Más al sur, los escasos ejemplos son de atribución cronocultural imprecisa o carecen de datos tafonómicos concluyentes.

La Cueva de La Pileta (Fig. 1) es un yacimiento emblemático para las manifestaciones simbólicas de las comunidades prehistóricas del sur de Iberia, en particular del Paleolítico Superior. Además, en 2015 se cumple el centenario de la primera pu-

\begin{tabular}{|c|c|c|c|}
\hline Yacimiento & $\mathbf{N}^{\mathbf{o}}$ & $\begin{array}{c}\text { Adscripción } \\
\text { cronocultural }\end{array}$ & Bibliografía \\
\hline Llonín & 1 & \multirow{8}{*}{ Magdaleniense } & \multirow{9}{*}{1} \\
\hline Ermitia & 1 & & \\
\hline Santimamiñe & 3 & & \\
\hline El Juyo & 2 & & \\
\hline El Pendo & 1 & & \\
\hline Tito Bustillo & 2 & & \\
\hline Abauntz & 1 & & \\
\hline Covarón & 1 & & \\
\hline Bolinkoba & 1 & Solutrense & \\
\hline Ardales & 1 & Sin contexto & 2 \\
\hline Malalmuerzo & 1 & Sin contexto & 3 \\
\hline La Pileta & 1 & Gravetiense & 4 \\
\hline
\end{tabular}

Tab. 1. Lámparas portátiles atribuidas al Paleolítico Superior halladas en España: 1. Rasilla et al. 2010 con referencias; 2. Cantalejo 1983; 3. Cantalejo et al. 2006. 4. Este trabajo.

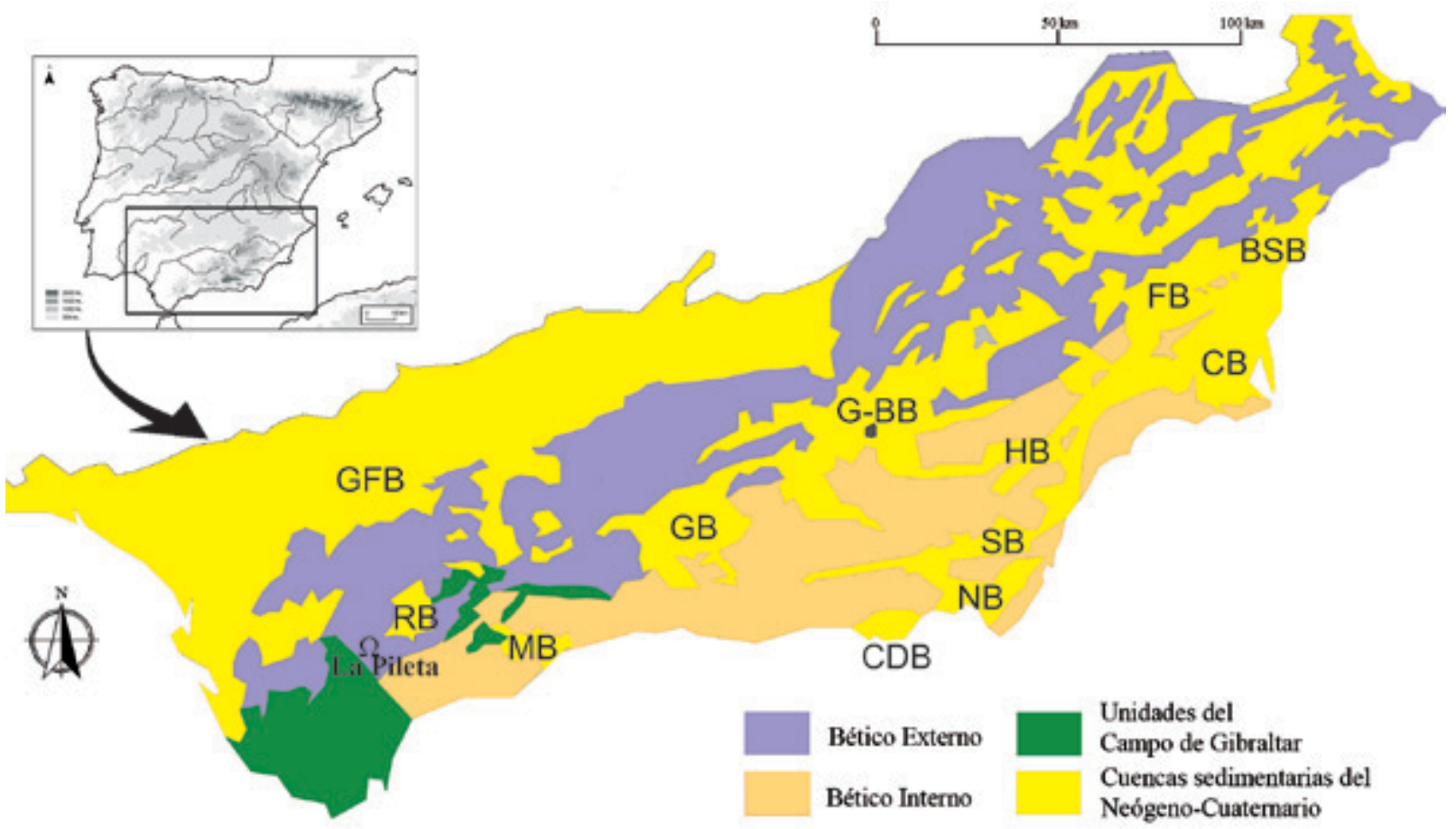

Fig. 1. Ubicación de cueva de La Pileta (Benaoján, Málaga) en relación a las cuencas neógenas del sur de Iberia. BSB (Bajo Segura), CB (Cartagena), CD (Campo de Dalías) FB (Fortuna), GB (Granada), G-BB (Guadix-Baza) GFB (Guadalquivir); HB (Huércal), MB (Málaga), NB (Níjar), RB (Ronda), SB (Sorbas).

Trab. Prehist., 73, N. ${ }^{\circ}$ 1, enero-junio 2016, pp. 115-127, ISSN: 0082-5638

doi: $10.3989 /$ tp.2016.12166 
blicación científica sobre la cavidad (Breuil et al. 1915). Con esa procedencia se encuentra depositado en el Museo de Málaga un objeto singular $\left(\mathrm{n}^{\mathrm{o}}\right.$ de inventario 232) que ha sido analizado de forma interdisciplinar y es un ejemplo de uno de los más antiguos hitos de iluminación portátil en el extremo sudoccidental de Europa.

\section{METODOLOGÍA}

La pieza 232 de La Pileta (Fig. 2) ha sido analizada siguiendo criterios paleontológicos, taxonómicos, arqueológicos y tafonómicos. Para su lectura se ha empleado una lupa binocular de hasta 120 aumentos y un microscopio digital Sony

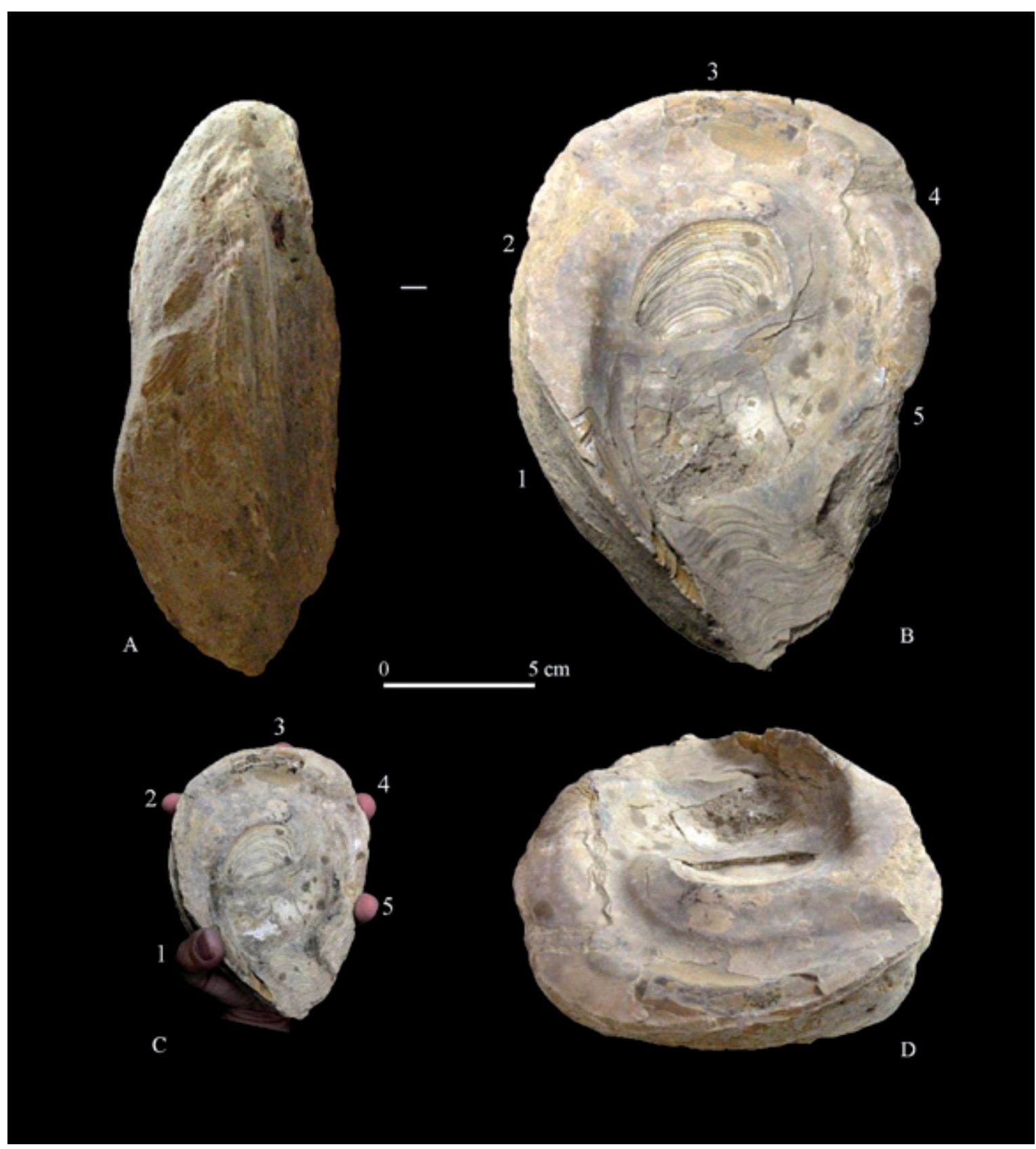

Fig. 2. Lámpara de La Pileta, Ostrea edulis fósil, orientada según su uso. 
interface DFW-X 700 con apoyo de iluminación de luz fría de tipo fibra óptica. La documentación gráfica se ha realizado con una cámara digital de 18 megapíxeles.

Las adherencias inorgánicas se han caracterizado mediante fluorescencia de rayos $\mathrm{X}$ portátil Oxford Instrument XMET-7500. Para la cuantificación se ha utilizado el programa calibrado de fábrica SOILS LE, basada en el método de parámetros fundamentales. Éste es el más adecuado para calcular la composición química cuando no existe otro basado en patrones primarios o se quiere analizar un gran número de elementos (Beckhoff et al. 2006: 403), como es nuestro caso. Recientemente se ha demostrado que, utilizando parámetros atómicos actualizados, se alcanzan errores ca. $1 \%$ para los elementos mayoritarios en materiales certificados (Elam et al. 2004), caso del citado programa SOILS LE.

El arte paleolítico de La Pileta se ha abordado según criterios tecnoestilísticos y de secuencia gráfica, también se ha tenido en cuenta para la discusión los datos sobre la arqueología del yacimiento (Cortés y Simón 2007) y el estado de la cuestión del Paleolítico Superior Inicial en el sur de Iberia.

\section{RESULTADOS}

La pieza 232 de La Pileta es una valva inferior de Ostrea edulis Linné, 1758 (Fig. 2). Formaba parte de un conjunto de materiales recogidos en superficie por Tomás Bullón, guarda de La Pileta, recabados por Juan Temboury (Delegado de Bellas Artes en Málaga) para integrar la colección fundacional del futuro Museo Arqueológico de Málaga. Este conjunto fue catalogado en 1939 por Simeón Giménez Reyna. Su estado de conservación es excelente. Es de gran talla (diámetros umbo-paleal de $178,81 \mathrm{~mm}$ y antero-posterior de $129,73 \mathrm{~mm})$, robusta, gruesa e inequilateral. Es una valva izquierda, aplanada, ligeramente convexa, oval-redondeada y alargada en dirección umbo-paleal. El umbo es pequeño y opistogiro. La valva conserva adherida en la cara dorsal (Fig. 3.3) una matriz arenosa con algún fragmento de esquisto que impide visualizar su ornamentación. En los bordes, lateralmente, se observan láminas concéntricas de crecimiento.
Su interior es liso y sólo se observan las impresiones musculares y del ligamento (Fig. 3.1). La charnela es disodonta, el ligamento interno muestra un área ligamentaria amplia y alargada $(48,12 \mathrm{~mm}$ de ancho y $47,37 \mathrm{~mm}$ de alto), formada por líneas longitudinales. El resilifer central está delimitado anterior y posteriormente por dos amplios bureletes (Fig. 3). Se observa anachomata en el margen lateral posterior. La impresión muscular única, de forma arriñonada, muestra unos márgenes laterales y ventral lisos.

Una serie de rasgos e indicios tafonómicos en la ostra permiten identificarla como fósil y articular una cadena operativa específica, así como identificar su funcionalidad:

1) Fase de captación: las características de la valva de ostra y la matriz conservada en la superficie dorsal se corresponden con los clásicos depósitos sedimentarios del Neógeno en la provincia de Málaga (Fig. 1).

2) Fase de configuración: en un lugar indeterminado se modificó puntualmente la valva para facilitar su uso:

a) Creación de un asidero más cómodo, mediante percusión directa con percutor duro y una técnica similar a la del retoque en industrias líticas talladas para desprender pequeños fragmentos de los márgenes y charnela (Figs. 2B-C y 4.5).

b) Desbastado y pulido posterior de la matriz sedimentaria adherida a la cara dorsal de la valva (Fig. 3.3 y 4.7, 11-13), que adopta una morfología de tendencia semiesférica (Fig. 3.2).

c) Creación, mediante repiqueteado, de una pequeña depresión en el ápice de la matriz arenosa (Fig. 4.7, 11-13). Su función pudo ser la ‘fijación' o estabilización de la lámpara sobre algún resalte del terreno (ápice de estalagmita, protuberancia, etc.) o calzo pues tiende a bascular hacia la parte distal de la valva, drenando el contenido del área paleal (Fig. 2D). La presencia de esta concavidad, el volumen y peso $(1,152 \mathrm{~g})$ de la ostra o la morfología de la cubeta de la lámpara apuntarían a su traslado apagada para facilitar la progresión por la caverna y su encendido una vez estabilizada.

d) Hay huellas del uso de filos cortantes, posiblemente de algún artefacto lítico tallado, quizás dejadas accidentalmente por el mismo instrumento con el que se regularizó la matriz sedimentaria, o vinculado con una actividad indeterminada (Fig. 3.1,12).

Trab. Prehist., 73, N. ${ }^{\circ}$ 1, enero-junio 2016, pp. 115-127, ISSN: 0082-5638

doi: $10.3989 /$ tp.2016.12166 


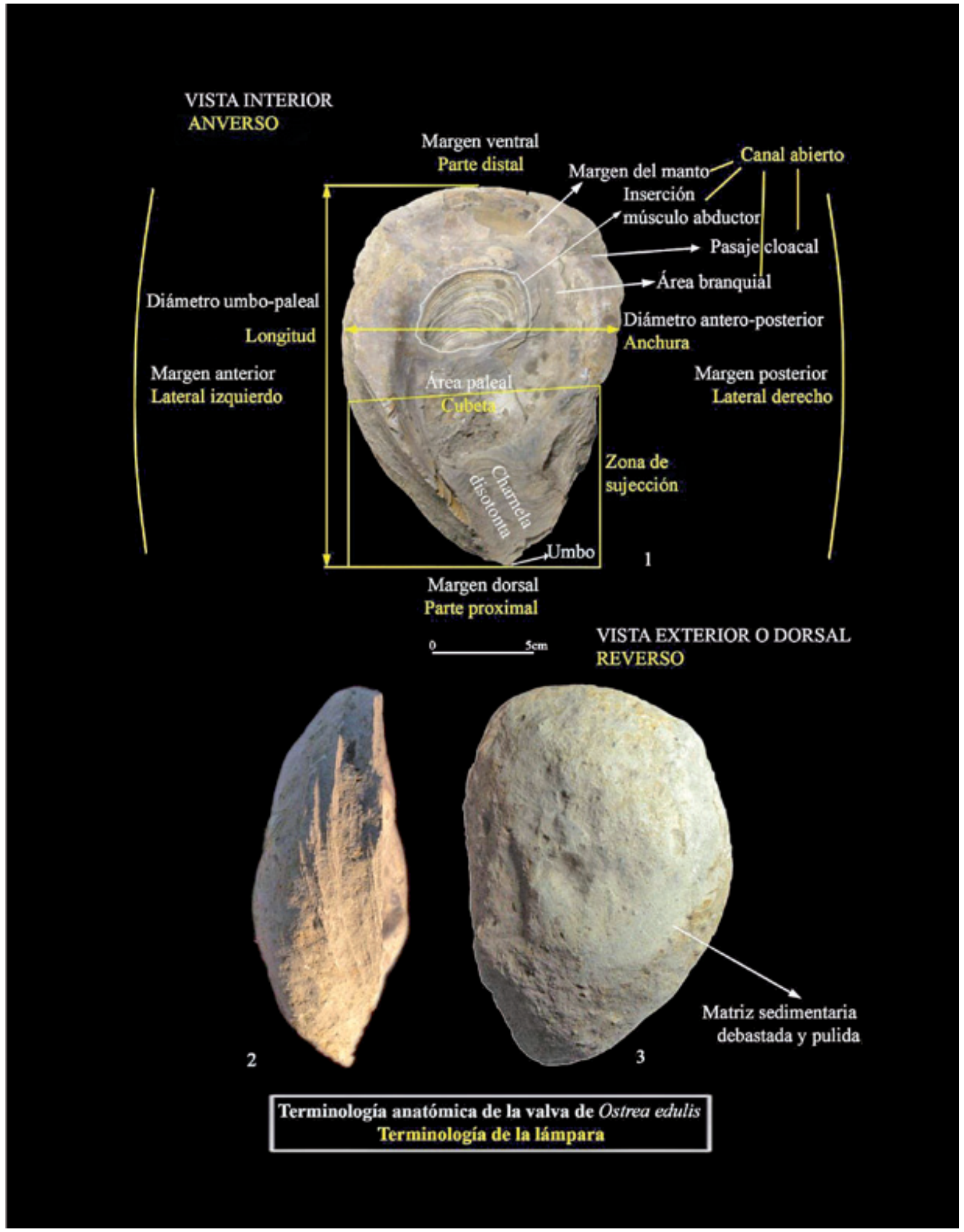

Fig. 3. Lámpara de La Pileta, Ostrea edulis fósil. Terminologías taxonómica y funcional empleadas. 


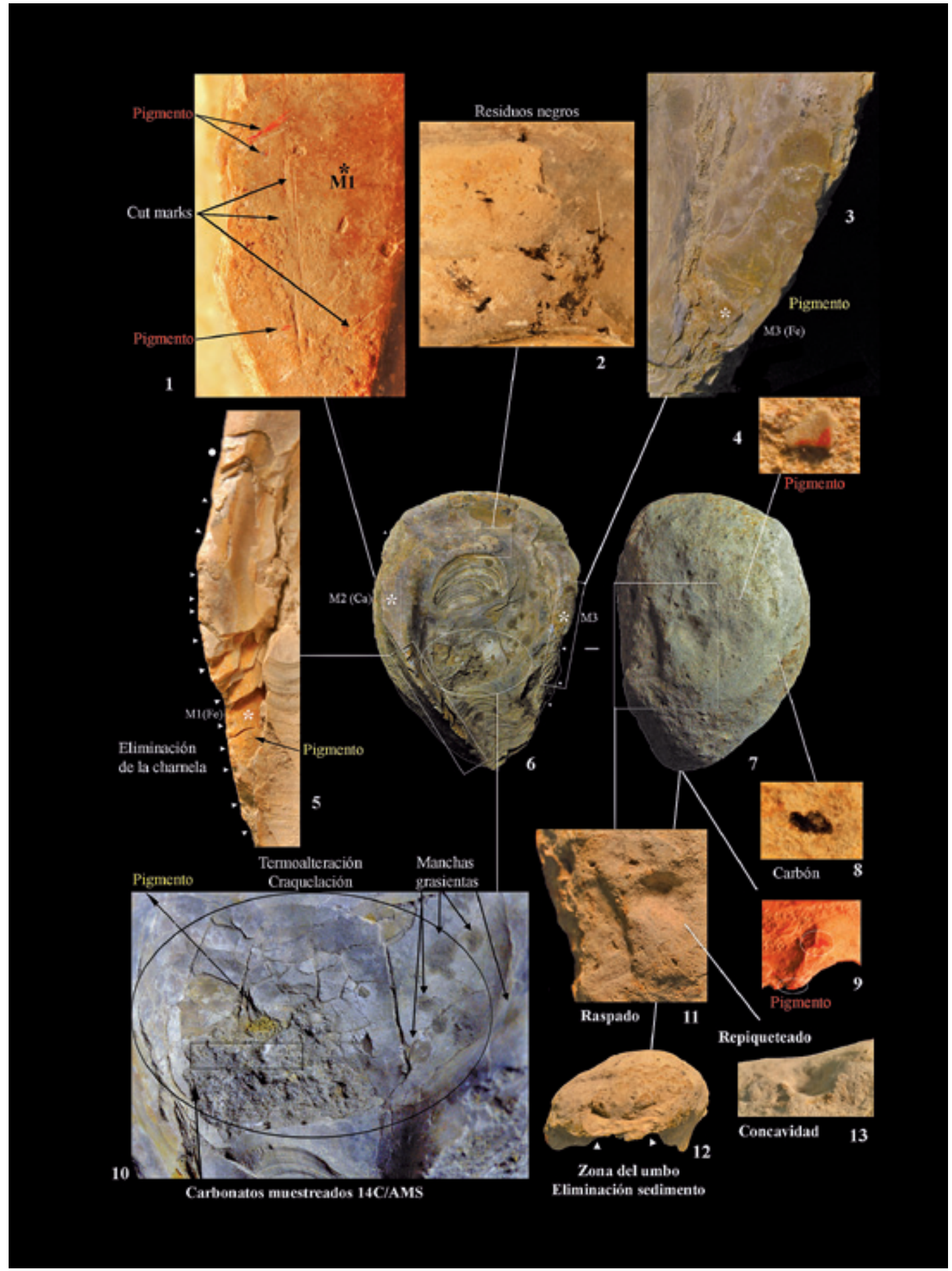

Fig. 4. Lámpara de La Pileta. Ostrea edulis fósil. Configuración: extracciones con percutor duro (puntos de impactos indicados mediante ) para eliminar la arista cortante de la charnela (5) y parte de la matriz sedimentaria (12), huellas de cut-marks (1), raspado (11), repiquetado (7, 11 y 13). Uso como lámpara: residuos de la mecha (2) y grasientos (10), termoalteración y craquelación (10). Uso de colorantes: amarillo (3, 5 y 10), rojo (1, 4 y 9) y carbón (8). Datación 14C/AMS (10). Muestreos mediante fluorescencia de rayos X: *M1 a M5.

Trab. Prehist., 73, N. ${ }^{\circ}$ 1, enero-junio 2016, pp. 115-127, ISSN: 0082-5638 doi: $10.3989 /$ tp. 2016.12166 
3) Fase de utilización como lámpara y durante la manipulación de pigmentos: todo el procesado de la valva iba encaminado a ese fin. El análisis permite identificar los siguientes episodios:

a) Encendido de algún tipo de mecha alimentada por un combustible, no identificado por el momento, entre los márgenes ventrales del área ligamentaria y de la impresión muscular (Fig. 3). La acción de la fuente de calor ocasionó la termoalteración y craquelación de la cara interna de la valva y una rubefacción suave de las zonas aledañas, que se va perdiendo a medida que nos alejamos de ella (Fig. 4.2,10).

b) La actividad de la lámpara dejó asimismo una serie de residuos en forma de trazas de carbón y manchas subcirculares de aspecto grasiento (Fig. 4.2,10). Estas últimas pudieran interpretarse también, en primera instancia, como residuos de un aglutinante de los que se solían añadir al pigmento (Balbín y Alcolea 2009). Sin embargo, su disposición y las características de los indicios tafonómicos de la pieza (vid infra) nos inducen a relacionarlas más probablemente con el chisporroteo de la llama en contacto con la materia grasa empleada como combustible.

c) Entre las actividades desarrolladas en el entorno de la lámpara está el uso de pigmento rojo, cuyas trazas aún son apreciables en distintas zonas de la valva (Fig. 4.1, 4, 9), y amarillo (Fig. 4.10). Este último es perceptible en el entorno de la zona que presumiblemente sirvió para la sujeción de la lámpara. Las manchas parecen así estar ligadas a la impregnación de los dedos con una sustancia amarillenta que fue trasferida al sujetar la concha (Fig. 4.3,5). Una veladura de carbonatos cubre las manchas grasientas y otros residuos lo que nos ha impedido, por el momento, analizar su composición por medios no destructivos. La pieza carece de indicios que permitan identificar su uso como molino (p.ej. pérdidas de materia por abrasionado o repiqueteados) o como recipiente para preparar/contener pigmentos (acumulaciones/impregnaciones de colorante en el área paleal/cubeta o termoalteración de la zona exterior o dorsal) entre otros. Por todo ello $\mathrm{y}$ en resumen, los datos tafonómicos permiten apuntar que la valva de ostra fue adaptada y utilizada como lámpara de canal abierto, empleando para ello el área en el que se situaban las branquias del animal, justo bajo el pasaje cloacal (Fig. 3). La cubeta de la lámpara (Fig. 4.6,10) estaría delimitada longitudinalmente por el margen ventral del área ligamentaria y el margen ventral de la impresión muscular $(86,31 \mathrm{~mm})$. Trasversalmente, queda definida esta cubeta por los márgenes laterales internos del área paleal $(75,60 \mathrm{~mm})$, siendo la profundidad medida desde el punto más profundo del área paleal hasta el plano axial de la valva de $28,09 \mathrm{~mm}$.

4) Fase 4 de abandono: la pieza quedó apoyada sobre la cara dorsal, en algún lugar donde se produjeron sucesivos episodios de goteo y evaporación de agua durante un periodo capaz de generar el depósito de carbonatos de $2 \mathrm{~cm}$ de espesor que recubre la cazoleta y la veladura de carbonatos de toda la cara interna de la concha. A tal efecto, la ostra debía estar estabilizada, dado que su tendencia natural es, como hemos expuesto, a bascular hacia la parte distal/ margen ventral (Fig. 2D), dado que el contenido de la cubeta se drena a través del área branquial hasta el pasaje cloacal y margen ventral de la ostra. Ello impediría la acumulación de agua en la cubeta/área paleal que generó el depósito de carbonatos y que ha dejado un levísimo reborde en la cubeta que dibuja el nivel alcanzado por el agua retenida. Así mismo, el que la pieza no tenga adheridos depósitos de carbonatos mucho más generalizados o potentes en la cubeta induce a pensar que se encontraba reservada en algún lugar de goteo esporádico de carácter ocasional y no junto a alguna zona de recrecimiento litoquímico muy activo. Estas deducciones sintonizarían con la idea generalizada de que las lámparas solían 'almacenarse' en lugares escogidos, a modo de escondrijos, al objeto de ser reutilizadas a lo largo del tiempo.

La carbonatación existente en la cazoleta ha sido muestreada con ayuda de una Dremmel empleada a baja revolución. Tras eliminar la capa más superficial, se extrajo una micromuestra que fue remitida para su datación mediante ${ }^{14} \mathrm{C} / \mathrm{AMS}$, obteniéndose una edad $26760 \pm 120$ BP (CNA$1660)$ o calibrada $(2 \sigma)$ comprendida entre 31775 31415 cal BP (Tab. 2). La edad corresponde a la medición del proceso de desintegración del $\mathrm{CO}_{2}$ existente en la atmósfera y que es fijado en el proceso de carbonatación.

$\mathrm{El}$ análisis mediante fluorescencia de rayos $\mathrm{X}$ se ha efectuado sobre cinco puntos de la superficie de la ostra y ha permitido identificar dos áreas con una coloración amarillenta muy nítida (Fig. 4.3, 5) 


\begin{tabular}{|c|c|c|c|c|c|c|}
\hline Atribución & Yacimiento & Nivel & BP & $\begin{array}{l}\text { AMS Cariaco- } \\
\text { Hulu BP } 2\end{array}$ & Laboratorio & Bibliografía \\
\hline \multirow{2}{*}{$\begin{array}{l}\text { Gravetiense } \\
\text { final }\end{array}$} & \multirow{9}{*}{ Vale Boi } & Talud/3 & $21830 \pm 195$ & $27152-25108$ & Wk-16415 & \multirow{9}{*}{1} \\
\hline & & Talud/3 & $22470 \pm 235$ & $30258-28222$ & Wk-13686 & \\
\hline \multirow{7}{*}{$\begin{array}{l}\text { Gravetiense } \\
\text { antiguo }\end{array}$} & & Terraza/4 & $24769 \pm 180$ & $30476-29120$ & Wk-24762 & \\
\hline & & Terraza/5 & $27720 \pm 370$ & $33097-31593$ & Wk-26081 & \\
\hline & & Terraza/3 & $24300 \pm 205$ & $29976-28158$ & Wk-12132 & \\
\hline & & Talud/3 & $23995 \pm 230$ & $24455-25535$ & Wk-16414 & \\
\hline & & Abrigo/D4 & $21896 \pm 186$ & $22268-21524$ & Wk-26803 & \\
\hline & & Abrigo/D4 & $28140 \pm 195$ & $33227-31943$ & Wk-31087 & \\
\hline & & Talud/3 & $24560 \pm 570$ & $25700-23420$ & Wk-26803 & \\
\hline \multirow{9}{*}{ Gravetiense } & \multirow{3}{*}{ Nerja } & NV10 & $24730 \pm 200$ & $30400-29160$ & GifA-102.023 & \multirow{3}{*}{2} \\
\hline & & NV11 & $24200 \pm 200$ & $29730-28410$ & Beta- 189080 & \\
\hline & & NV13 & $24480 \pm 110$ & $30100-28940$ & Beta-131576 & \\
\hline & Bajondillo & 10 & $24344 \pm 2653^{*}$ & - & MAD-2470 & 3 \\
\hline & La Pileta & - & $26760 \pm 120$ & $31775-31415$ & CNA-1660 & 4 \\
\hline & \multirow{8}{*}{$\begin{array}{c}\text { Gorham/ } \\
\text { Ext** }\end{array}$} & $\mathrm{D}$ & $27860 \pm 300$ & $33117-31717$ & GRN-1363 & \multirow{2}{*}{5} \\
\hline & & $\mathrm{D}$ & $29700 \pm 200$ & $33970-33184$ & GRN-1455 & \\
\hline & & Contexto-7 & $25680 \pm 280$ & $31599-29887$ & Ox A-6997 & \multirow{6}{*}{6} \\
\hline & & Contexto-15 & $28680 \pm 240$ & $34003-32303$ & Ox A-7792 & \\
\hline \multirow{4}{*}{$\begin{array}{l}\text { Paleolítico } \\
\text { Superior } \\
\text { Inicial }\end{array}$} & & CHm.5/Contexto-9 & $30200 \pm 200$ & $34724-34056$ & Ox A-7074 & \\
\hline & & CHm.5/Contexto-9 & $29800 \pm 700$ & $35203-32734$ & Ox A-7075 & \\
\hline & & CHm.5/Contexto-9 & $30250 \pm 700$ & 35916-33124 & Ox A-7076 & \\
\hline & & CHm.5/Contexto-9 & $29250 \pm 650$ & $34732-33412$ & Ox A-7077 & \\
\hline \multirow{2}{*}{ Auriñaciense } & \multirow{3}{*}{ Bajondillo } & 11 & $33690 \pm 1195$ & $37500 \pm 1541$ & Ua-17150 & \multirow{3}{*}{3} \\
\hline & & 11 & $32770 \pm 1065$ & $37500 \pm 1541$ & Ua-18050 & \\
\hline $\begin{array}{l}\text { Paleolítico } \\
\text { Superior } \\
\text { Inicial }\end{array}$ & & 13 & $37005 \pm 1790$ & $41232 \pm 1787$ & Ua-18270 & \\
\hline
\end{tabular}

Tab. 2. La fecha AMS de la lámpara de La Pileta en relación a las dataciones disponibles para el Paleolítico Superior Inicial en el sur de Iberia.

*TL, **Cemented hearths member=nivel D de Waechter, según Pettitt y Bailey 2000. 1. Bicho et al. 2010; 2. Aura et al. 2010 con referencias. 3. Cortés 2007a. 4. Este trabajo. 5. Waechter 1964. 6. Pettitt y Bailey 2000.

que presentan un gran contenido en hierro $(>95 \%$ at.), probablemente óxido de hierro (M3-4, Tab. 3 y Fig. 4.3,5), datos que contrastan con la composición calcítica de la concha $\left(\mathrm{CaCO}_{3}\right)(\mathrm{M} 1, \mathrm{Tab} .3 \mathrm{y}$ Fig. 4.1). Un tercer punto corresponde a una fina película de carbonatos cálcico-magnésicos (M2,5,
Tab. 3 y Fig. 4.2,10). Los trazos de coloración rojiza distribuidos en distintas áreas de la lámpara no han podido ser analizados. Al contrario de lo constatado en el ocre amarillo (semilíquido), las trazas dejadas por el pigmento rojo parecen evidenciar un estado sólido (Fig. 4.1). 


\begin{tabular}{|c|c|c|c|c|c|c|c|c|c|c|c|}
\hline $\mathbf{N}^{\mathbf{0}}$ & $\mathbf{M g}$ & $\mathbf{A l}$ & $\mathbf{S i}$ & $\mathbf{P}$ & $\mathbf{S}$ & $\mathbf{C l}$ & $\mathbf{K}$ & $\mathbf{C a}$ & $\mathbf{T i}$ & $\mathbf{M n}$ & $\mathbf{F e}$ \\
\hline M1 & - & $16^{\prime} 64$ & $23^{\prime} 24$ & $0^{\prime} 00$ & $21^{\prime} 36$ & $0^{\prime} 54$ & - & $38^{\prime} 00$ & $0^{\prime} 01$ & - & $0^{\prime} 18$ \\
\hline M2 & $22^{\prime} 52$ & $16^{\prime} 04$ & $17^{\prime} 43$ & $0^{\prime} 05$ & $17^{\prime} 06$ & $0^{\prime} 40$ & - & $26^{\prime} 32$ & $0^{\prime} 01$ & $0^{\prime} 01$ & $0^{\prime} 14$ \\
\hline M3 & - & $0^{\prime} 32$ & $0^{\prime} 50$ & - & $0^{\prime} 29$ & $0^{\prime} 01$ & $0^{\prime} 01$ & $0^{\prime} 45$ & - & - & $98^{\prime} 42$ \\
\hline M4 & - & $0^{\prime} 15$ & $0^{\prime} 26$ & - & $0^{\prime} 06$ & - & - & $0^{\prime} 18$ & - & - & $99^{\prime} 33$ \\
\hline M5 & - & $20^{\prime} 13$ & $32^{\prime} 93$ & $0^{\prime} 08$ & $19^{\prime} 20$ & $0^{\prime} 51$ & $0^{\prime} 28$ & $26^{\prime} 58$ & $0^{\prime} 04$ & $0^{\prime} 02$ & $0^{\prime} 19$ \\
\hline
\end{tabular}

Tab. 3. Composición elemental expresada en porcentaje atómico de cinco puntos de la superficie de la ostra, localizados en la figura 2.1,3,5,10 (*M1 a 5). Datos obtenidos por fluorescencia de rayos X.

\section{DISCUSIÓN}

\subsection{Lámpara}

La ostra analizada procede de un depósito fosilífero Neógeno. Aunque no es descartable un origen en niveles pliocenos ubicados en la costa de Málaga (Fig. 1), estos fósiles están también con frecuencia asociados a niveles miocenos. La cuenca sedimentaria fosilífera más cercana (a unos $10 \mathrm{~km}$ ) es la de Ronda (Fig. 1). La cara interna de la ostra no muestra indicios de erosión vinculados al transporte en medio hídrico o a fracturas mecánicas naturales, producidas por desplazamiento gravitacional, ni tampoco indicios de erosión subaérea. En consecuencia, es probable que la pieza fuera recogida en el propio depósito fosilífero o en sus inmediaciones, antes de que aquellos procesos dejaran una huella nítida sobre la concha. Por tanto, podemos postular que la pieza fue transportada, como mínimo, unos $10 \mathrm{~km}$.

La identificación funcional como lámpara se basa en el escrutinio al que ha sido sometida la valva respecto a los requisitos requeridos para ser considerada como tal (Beaune 1987: 12, 151-152). Así, la ostra dispone de una marcada concavidad o cubeta, a la que se circunscriben inequívocos indicios de termoalteración (Figs. 2D y 4.10). Existen residuos orgánicos en la zona activa y bordes de la pieza (Fig. 4.10) y contamos con la adecuación intencional de la valva mediante extracciones con percutor duro para facilitar la sujeción (Figs. 2C y 4.5).

Las lámparas paleolíticas son artefactos poco frecuentes. Para todo el Paleolítico Superior en Francia, apenas se superan los 300 ejemplares, en tanto que en la Península Ibérica sólo tenemos inventariadas 15 piezas (Tab. 1). Las lámparas francesas muestran dos grandes agrupaciones cronoculturales: en el Magdaleniense $(71,8 \%)$ y en el Paleolítico Superior Inicial (13,1\%) (Beaune 1987: 36), en especial en el Gravetiense, con el que encajaría la fecha del ejemplar de La Pileta.

Beaune (1987) cataloga la recolección y uso de fósiles o fragmentos de roca conservando fósiles visibles: un Pecten en Trois Frères, cuatro ostras (Badegoule, Flageolet, Jaurias y Moulin-Neuf) y un molusco, no determinado taxonómicamente, en Liveire. Los corales aparecen en cuatro lugares (Le Puy-de-Lacan, Laussel, Richard y, posiblemente, Teyjat), junto con casos sin asignación taxonómica (p.ej. Vidon con un gran fósil en el reverso o el molde de un gran bivalvo en un ejemplar de Enlène 1). Además aparecen fósiles de menor tamaño, visibles en superficie, en cinco piezas (Flageolet I, Labatut y dos de La Madeleine y otra en Jaurias). El inventario asciende así a 18 objetos $(5,96 \%)$ de los analizados por Beaune (1987). Más interesante es el porcentaje de yacimientos donde hay alguna pieza con estas características. Así, en 17 (ca. 19\%) de los 195 emplazamientos citados por Beaune (1987) se eligen soportes con fósiles, lo que resulta más significativo. Si añadimos también los elementos geológicamente exóticos, como geodas y minerales y rocas vistosos (esteatita, areniscas, etc.), el número de piezas crece significativamente. En este contexto, parece obvio que las características de cualquier concavidad natural (p.ej. cavidades medulares u órbitas) son idóneas para servir de lámpara, aunque a la hora de escoger la materia prima para este tipo de artefacto probablemente participasen criterios vinculados con la singula- 
ridad y el exotismo, como la presencia de "seres vivos petrificados" en las rocas.

Existe cierto consenso sobre la frecuente asociación de las lámparas paleolíticas con ámbitos con una fuerte carga simbólica y cavidades con manifestaciones artísticas paleolíticas (Rasilla et al. 2010), circunstancias que concurren en La Pileta. En resumen, parece probable que la pieza de La Pileta se empleara para tareas de iluminación de las zonas interiores y que, por los indicios de manipulación de colorantes, quizás estuviera asimismo vinculada con los primeros momentos de expresión simbólica representados en la cavidad (fase preparatoria, ejecución, visualización, etc.).

En el contexto del sur de Iberia, las lámparas atribuidas al Paleolítico son fijas y portátiles. La existencia de las primeras se ha propuesto en Ardales, La Pileta y Nerja (Cantalejo et al. 2006; Medina et al. 2012); y para las segundas, un ejemplar en Malalmuerzo y otro en Ardales (Cantalejo 1983; Cantalejo et al. 2006). Más incertidumbre suscitan las valvas de Pecten documentadas en Navarro y Nerja (Medina et al. 2012), debido a que no detallan la caracterización tafonómica que conduce a tal identificación.
El segmento cronocultural al que se puede atribuir el uso de la lámpara de La Pileta se encuentra dentro de la problemática del tránsito Paleolítico Medio-Superior en el sur de Iberia situado, hasta hace poco, en torno a $30 \mathrm{cal} \mathrm{ka} \mathrm{BP.}$ Esta edad haría factible que la ostra fósil hubiese incluso podido ser recolectada, transformada y usada como lámpara por los neandertales, cuyos vestigios, recordémoslo, también están presentes en el yacimiento (Cortés y Simón 2007). En este sentido, la recogida de fósiles o el empleo de pigmentos parecen suficientemente documentados entre los neandertales (vid. p.ej. Soressi y d'Errico 2007; Zilhão et al. 2010), aunque ninguna de estas prácticas ha sido constatada aún entre los del sur de Iberia.

Los argumentos más sólidos para descartar la asignación de la lámpara de La Pileta al Paleolítico Medio los encontramos en cuatro conjuntos de datos:

La revisión de las cronologías tardías para el Paleolítico Medio en el sur de Iberia, que apuntan a unas cronologías $>39$ cal ka BP para los momentos finales, en sintonía con los expresado en Europa occidental (Higham et al. 2014).

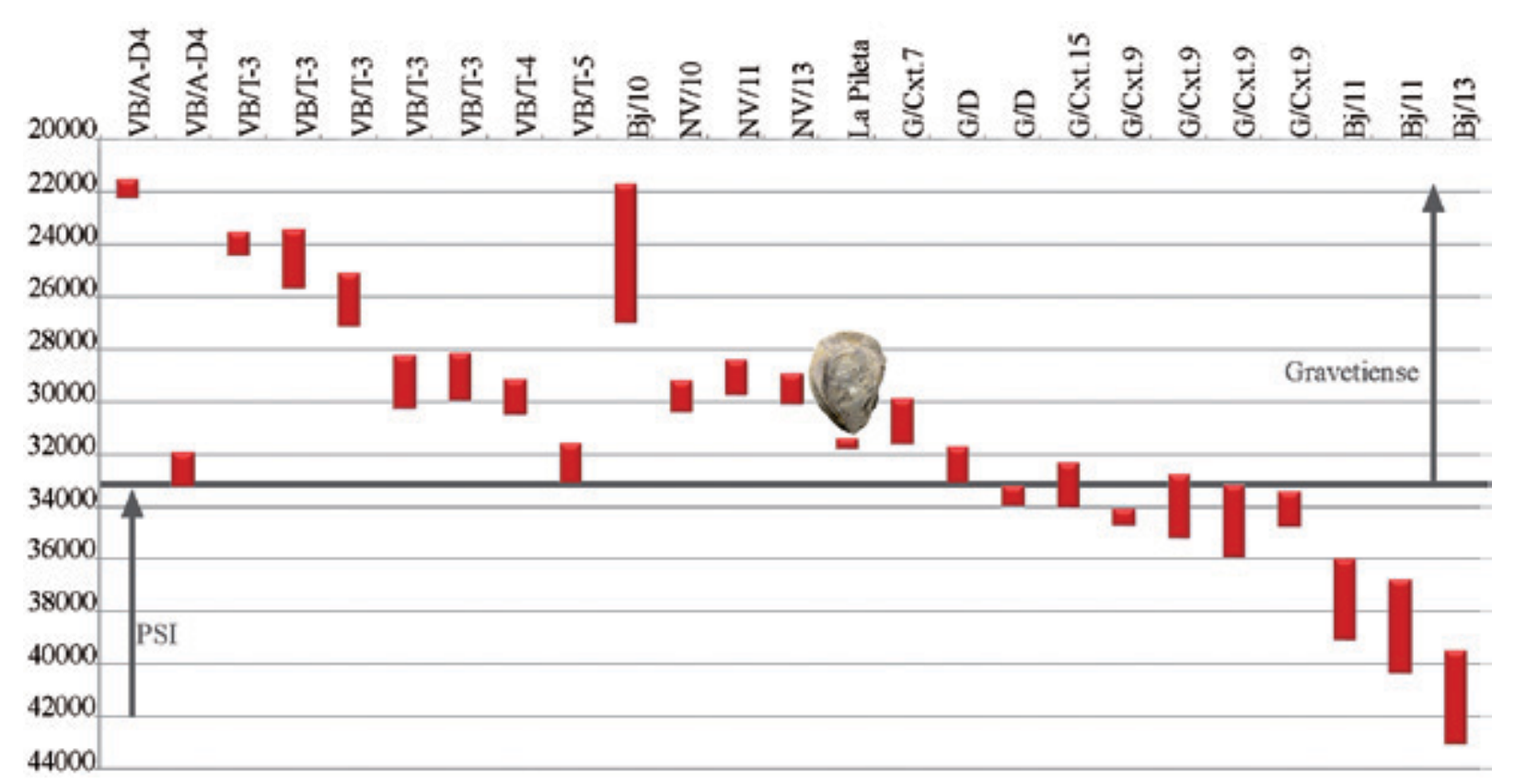

Fig. 5. Fecha AMS cal B.P. de la lámpara de La Pileta en relación a las dataciones disponibles para el Paleolítico Superior Inicial en el sur de Iberia (datos numéricos y bibliografía en tabla 2). Bj (Bajondillo); G (Gorham); NV (Nerja); VB (Vale Boi); PSI (Paleolítico Superior Inicial).

Trab. Prehist., 73, N. ${ }^{\circ}$ 1, enero-junio 2016, pp. 115-127, ISSN: 0082-5638 doi: $10.3989 /$ tp.2016.12166 
La consolidación de la secuencia cronocultural del Paleolítico Superior Inicial de la región, en especial para el Gravetiense (Cortés 2007 b; Cortés et al. 2012),

La datación ${ }^{14} \mathrm{C} / \mathrm{AMS}$ directa del carbonato de la cazoleta de la lámpara de La Pileta, que coincide con el rango cronológico del Gravetiense, al menos, en tres yacimientos del sur de Iberia (Fig. 5, Tab. 2). Aún así, habría que considerar la fecha como una edad mínima, relacionada con el depósito del carbonato. Éste pudo, posiblemente, haber sido contemporáneo a la amortización de la lámpara, aunque tampoco podemos descartar que, entre ambos episodios, existiese un lapso de tiempo -no necesariamente dilatado- imposible de evaluar con los datos disponibles (para poder responder a esta pregunta habría que intentar datar la materia orgánica conservada en la cazoleta de la lámpara).

Casi la totalidad de las lámparas pleistocenas caracterizadas en el occidente de Europa aparecen en el Paleolítico Superior estando vinculadas, en muchos casos, con actividades de iluminación en cuevas con arte.

En resumen, los datos disponibles apuntan a que la lámpara de La Pileta fue empleada por humanos modernos durante el Gravetiense.

\subsection{Arte presolutrense}

Los indicios de colorantes presentes en la lámpara de La Pileta permiten una reflexión sobre el arte paleolítico de La Pileta, aunque para poder correlacionar los pigmentos presentes en la ostra con los de las pinturas de la cueva sería necesario analizarlos y compararlos, algo inviable por el momento. No obstante, resulta sugerente la posibilidad de vincular la lámpara con la ejecución de uno de los primeros horizontes gráficos de La Pileta en contextos gravetienses. En este sentido, si el Gravetiense parece que constituye el momento de consolidación del poblamiento de las comunidades de humanos modernos y del Paleolítico Superior en el sur de Iberia (Cortes et al. 2012), se antoja lógico pensar que estas comunidades traen consigo, además de las industrias líticas talladas características, buena parte del acervo cultural que las caracteriza en otras regiones y no sólo los artefactos líticos tallados de este tecnocomplejo.
En tal sentido, el mundo simbólico Gravetiense en este área cuenta con pruebas de manufactura y uso de colorantes y adornos en Nerja (Aura et al. 2010) y Vale Boi, este último también con ejemplos inéditos de arte mobiliar (Bicho et al. 2010: 229), o la aquí tratada recolección y uso de fósiles en La Pileta, por lo que resultaría extraña la ausencia de grafismo rupestre. Así pues, en ausencia de dataciones de los vestigios gráficos parietales de La Pileta, deberemos recurrir a criterios tecnoestilísticos.

En este último sentido cabe reseñar que el horizonte artístico más antiguo de La Pileta ha sido, por lo general, atribuido al Solutrense (Sanchidrián 1997), aunque diversos autores han propuesto la existencia de un horizonte previo. El primero fue Breuil (Breuil et al. 1915) quien encuadraba su "horizonte de serpentiformes amarillos" (Galerías de las Cabras y de las Serpientes) en el "ciclo auriñacoperigordiense". Con posterioridad, Fortea (2005), en el marco de una revisión del arte presolutrense ibérico, identifica un horizonte amarillo de trazos digitales y pigmentos pastosos con los que se realizan algunos zoomorfos (p.ej. cabras y rinoceronte) y determinados meandriformes. Complementa la lectura del panel del tramo final de la Galería de las $\mathrm{Ca}$ bras, reseñando un conjunto de manos en positivo que no habían sido valoradas. Por último, pone de manifiesto sus paralelismos en yacimientos del Mediterráneo francés, como Baume-Latrone (Francia), asociados también a manos en positivo que en la actualidad se encuadran dentro del Gravetiense.

Los serpentiformes ejecutados con pigmentos amarillos y con una técnica tan particular en contextos anteriores al Solutrense ya fueron apuntados por Breuil (Breuil et al. 1915), al comentar el paralelismo entre La Pileta, Gargas y Hornos de la Peña. Motivos afines aparecen también en BaumeLatrone o Llonín (Fortea 2005). A estos ejemplos cabe añadir los bloques grabados con este tipo de motivos y cúpulas del Abri Pataud recuperados en contextos del Gravetiense medio (Jaubert 2008). Todo ello sin olvidar que la lámpara de La Pileta creemos que demuestra la manipulación contemporánea de colorantes de distinto cromatismo en el Gravetiense. Ello nos induce a ser cautos a la hora de reducir los horizontes gráficos antiguos de La Pileta al pigmento amarillo o de recurrir a superposiciones de diferente color, sin una ico- 
nografía definida, para distinguir los horizontes gráficos de La Pileta.

En resumen, la edad y los indicios de los pigmentos asociados a la lámpara analizada podrían apoyar los criterios técnicos y cronoestilísticos propuestos por Breuil y Fortea.

\section{CONCLUSIONES}

Los datos taxonómicos y tafonómicos obtenidos en el análisis de la valva de ostra ponen de manifiesto que se trata de un ejemplar fósil, recolectado a una distancia de $>10 \mathrm{~km}$.

Los indicios y microvestigios conservados (alteraciones térmicas, residuos de la mecha y el combustible, Fig. 4.2 y 10) permiten identificar su uso como lámpara. También indican la utilización de colorantes (rojo, amarillo y, quizás, negro, Fig. 4.8) en el entorno inmediato de la lámpara. El óxido de hierro amarillo (Fig. 4.3, 5 y 10) se encontraba diluido e impregnaba los dedos y mano de alguno de los usuarios, quedando transferido a la lámpara en las zonas de sujeción, mientras que el colorante rojizo dejó una traza propia de una forma sólida (Fig. 4.1). Estas evidencias sugieren que la lámpara estuviera vinculada a la preparación de pigmentos y/o a la aplicación de los mismos sobre las paredes de la cueva, aspectos sobre los que resulta imposible profundizar por el momento.

Tiempo después de ser apagada la lámpara y abandonada en superficie, la cubeta quedó sellada por una costra carbonatada (Fig. 4.6,10), cuya edad está encuadrada en el desarrollo del Gravetiense antiguo regional. En este sentido, se trata del elemento de iluminación portátil con datación directa más antiguo conocido para Iberia (Tab. 2).

La fecha AMS obtenida parece confirmar la frecuentación humana de La Pileta durante el Paleolítico Superior Inicial, como apuntaban ya algunos indicios tecnológicos (Cortés y Simón 2007) y abre una nueva dimensión en la comprensión de la ocupación y uso de La Pileta durante este periodo.

\section{AGRADECIMIENTOS}

Este trabajo es una contribución a los proyectos de investigación HAR2013-44269-P (Ministerio de
Economía y Competividad), "Grupo HUM-949. Tellus. Prehistoria y Arqueología en el sur de Iberia, Interdisciplinary Center for Archaeology and the Evolution of Human Behaviour" (Universidade do Algarve, Portugal), "Estudio de materiales procedentes de la Cueva de La Pileta de Benaoján depositados en el Museo de Málaga" (IDPH/ JT/18/05/PU/MA), autorizado por la Consejería de Cultura de la Junta de Andalucía a uno de los autores (MCS). A la familia Bullón agradecemos sus atenciones e información inédita sobre La Pileta.

\section{BIBLIOGRAFÍA}

Aura, J. E.; Jordá, J. F.; Pérez, M.; Badal, E.; Morales, J. V.; Avezuela, B.; Tiffagom, M. y Jardón, P. 2010: "Treinta años de investigación sobre el Paleolítico superior en Andalucía: la Cueva de Nerja (Málaga, España)". En X. Mangado (ed.): El Paleolítico superior peninsular. Novedades del siglo XXI. Homenaje a Javier Fortea. Universidad de Barcelona. Barcelona: 149-172.

Balbin, R. y Alcolea, J. J. 2009: "Les colorants de l'art paléolithique dans les grottes et en plein air". L'Anthropologie 113: 559-601.

Beaune, S.A. de 1987: Lampes et godets au Paléolithique. Gallia Préhistoire XXIII supplément. CNRS. Paris.

Beckhoff, B.; Kanngießer, B.; Langhoff, N.; Wedell, R. y Wolff, H. 2006: Handbook of practical X-ray fluorescence analysis. Berlín, Springer.

Bicho, N.; Manne, T.; Cascalheira, C.; Mendonça, C.; Évora, M.; Gibaja. J. F. y Pereira, T. 2010: Paleolítico Superior do sudoeste da Península Ibérica: o caso do Algarve. En X. Mangado (ed.): El Paleolítico superior peninsular. Novedades del siglo XXI. Homenaje a Javier Fortea. Universidad de Barcelona. Barcelona: 219-238.

Breuil, H.; Obermaier, H. y Verner, W. 1915: La Pileta à Benaoján (Málaga, Espagne). Institut de Paléontologie Humaine. Mónaco.

Cantalejo, P. 1983: "La cueva de Malalmuerzo (Moclín, Granada): una nueva estación con arte rupestre paleolítico en el área mediterránea". Antropología y Paleoecología Humana 3: 59-100.

Cantalejo, P.; Maura, R.; Espejo, M. M.; Ramos, J.; Medianero, J.; Aranda, A. y Durán, J. J. 2006: La Cueva de Ardales: Arte prehistórico y ocupación en el Paleolítico Superior. Estudios, 1985-2005. Centro de Ediciones de la Diputación de Málaga. Málaga.

Cortés Sánchez, M. (ed.) 2007a: Cueva de Bajondillo (Torremolinos). Secuencia cronocultural y paleoambiental del Cuaternario reciente en la Bahía

Trab. Prehist., 73, N. ${ }^{\circ}$ 1, enero-junio 2016, pp. 115-127, ISSN: 0082-5638

doi: $10.3989 /$ tp.2016.12166 
de Málaga. Centro de Ediciones de la Diputación de Málaga. Málaga.

Cortés Sánchez, M. 2007b: El Paleolítico Medio y Superior en el sector central de Andalucía (Córdoba y Málaga). Museo de Altamira, Monografías 22, Ministerio de Cultura. Madrid.

Cortés Sánchez, M.; Marreiros, J.; Simón Vallejo, M. D.; Gibaja Bao, J. F. y Bicho, N. F. 2012: "Reevaluación del Gravetiense en el sur de Iberia". En C. de las Heras Martín, J. A. Lasheras Corruchaga, Á. Arrizabalaga Valbuena, M. de la Rasilla Vives (eds.): Pensando el Gravetiense: nuevos datos para la región cantábrica en su contexto peninsular y pirenaico. Monografías del Museo Nacional y Centro de Investigación de Altamira 23, Ministerio de Cultura. Madrid: 73-85.

Cortés Sánchez, M. y Simón Vallejo, M. D. 2007: “La Pileta (Benaoján, Málaga) cien años después. Aportaciones al conocimiento de su secuencia arqueológica". Saguntum 40: 45-64.

Elam, W.; Shen, R.; Scruggs, B. y Nicolosi, J. 2004: "Accuracy of standardless FP analysis of bulk and thin film samples using a new atomic database". Advances in X-ray Analysis 47: 104-109.

Fernández Peris, J.; Barciela González, V.; Blasco, R.; Cuartero, F.; Fluck, H.; Sañudo, P. y Verdasco, C. 2012: "The earliest evidence of hearths in Southern Europe: The case of Bolomor Cave (Valencia, Spain)". Quaternary International 247: 267-277.

Fortea, J. 2005: "La plus ancienne production artistique du Paléolithique ibérique”. En A. Broglio y G. Dalmeri (eds.): Pitture paleolitiche nelle Prealpi venete: Grotta di Fumane e Riparo Dalmieri. Memorie del Museo civico di storia naturale di Verone. II Serie, Sezione Scienze dell'uomo, 9: 89-99. Verona, Museo civico di storia naturale.

Higham, T.; Douka, K.; Wood, R.; Ramsey, C. B.; Brock, F.; Basell, L.; Camps, M.; Arrizabalaga, A.; Baena, J.; Barroso-Ruiz, C.; Bergman, C.; Boitard, C.; Boscato, P.; Caparros, M.; Conard, N. J.; Draily, C.; Froment, A.; Galvan, B.; Gambassini, P.; Garcia-Moreno, A.; Grimaldi, S.; Haesaerts, P.; Holt, B.; Iriarte-Chiapusso, M. J.; Jelinek, A.; Jorda Pardo, J. F.; Maillo-Fernandez, J.M.; Marom, A.; Maroto, J.; Menendez, M.; Metz, L.; Morin, E.; Moroni, A.; Negrino, F.; Panagopoulou, E.; Peresani, M.; Pirson, S.; de la Rasilla, M.; Riel-Salvatore, J.; Ronchitelli, A.M.; Santamaria, D.; Semal, P.; Slimak, L.; Soler, J.; Soler, N.; Villaluenga, A.; Pinhasi, R. y Jacobi, R. 2014: "The timing and spatiotemporal patterning of Neanderthal disappearance". Nature 512, 7514: 306-309.

Jaubert, J. 2008: "L'Art pariétal gravettien en France: éléments pour un bilan chronologique". Paléo 20: 439-474.
Medina, M. A.; Cristo, A.; Romero, A. y Sanchidrián, J. L. 2012: “Otro punto de luz. Iluminación estática en los 'santuarios' paleolíticos: el ejemplo de la Cueva de Nerja (Málaga, España)”. En J. Clottes (ed.): L'art pléistocène dans le monde. Actes du Congrès IFRAO (Tarascon-sur-Ariège 2010), Symposium Art pléistocène en Europe. $\mathrm{N}^{\circ}$ spécial de Préhistoire, Art et Sociétés, Bulletin de la Société Préhistorique Ariège-Pyrénées LXV-LXVI: 105-121.

Monge Gómez, M. G.; Carretero León, M. I.; Pozo Rodríguez, M. y Barroso Ruiz, C. 2014: "Mineralogical changes in fossil bone from cueva del Ángel, Spain: Archaeological implications and ocurrence of whitlockite". Journal of Archaeological Science 46: 6-15.

Pettitt, P. B. y Bailey, R. M. 2000: “AMS Radiocarbon and Luminiscence dating of Gorham's and Vanguard Cave, Gibraltar, and implications for the Middle to Upper Palaeolithic Transition in Iberia". En C. B. Stringer, R. N. E. Barton y J. C. Finlayson (eds.): Neanderthals on the Edge. Oxbow Books. Oxford: 155-162.

Rasilla, M. de la; Duarte, E.; Santamaría, D.; Martínez Fernández, J.; Fernández de la Vega, J.; Rodríguez Otero, V. y Fortea, J. 2010: "Licnología paleolítica: las lámparas de las cuevas de Llonín y El Covarón (Asturias)". Zephyrus LXV: 103-116.

Sanchidrián, J. L. 1997: "Propuesta de la secuencia figurativa en la Cueva de La Pileta”. En J. M. Fullola y N. Soler (eds.): El món mediterrani després del Pleniglacial (18.000-12.000 B.P.). Sèrie Monogràfica 17, Centre d'Investigacions Arqueològiques, Museo d'Arqueologia de Catalunya. Gerona: 411-430.

Soressi, M. y d'Errico, F. 2007: "Pigment, gravures, parures: les comportements symboliques controversés des Néandertaliens". En B. Vandermeersch y B. Maureille (eds.): Les Néandertaliens. Biologie et cultures. Comité des travaux historiques et scientifiques. Paris: 297-309.

Waechter, J. D'A 1964: "The excavations at Gorham's Cave, Gibraltar, 1951-1954”. Bulletin Institute of Archaeology of London 4: 189-221.

Zilhão, Z.; Angelucci, D. E.; Badal-García, E.; d'Errico, F.; Daniel, F.; Dayet, L.; Douka, K.; Higham, T. F. G.; Martínez-Sánchez, M. J.; MontesBernárdez, R.; Murcia-Mascarós, S.; Pérez-Sirvent, C.; Roldán-García, C.; Vanhaeren, M.; Villaverde, V.; Wood, R. y Zapata, J. 2010: "Symbolic use of marine shells and mineral pigments by Iberian Neandertals". Proceedings of the National Academy of Sciences of the United States of America 107, 3: 1023-1028. 